\title{
Pressure-induced dimerization and valence bond crystal formation in the Kitaev-Heisenberg magnet $\alpha-\mathrm{RuCl}_{3}$
}

\author{
G. Bastien, ${ }^{1, *}$ G. Garbarino, ${ }^{2}$ R. Yadav,${ }^{1}$ F. J. Martinez-Casado, ${ }^{3,4}$ R. Beltrán Rodríguez, ${ }^{1}$ Q. Stahl, ${ }^{5}$ M. Kusch, ${ }^{5}$ \\ S. P. Limandri, ${ }^{6}$ R. Ray, ${ }^{1,7}$ P. Lampen-Kelley, ${ }^{8,9}$ D. G. Mandrus, ${ }^{8,9}$ S. E. Nagler, ${ }^{10}$ M. Roslova, ${ }^{11}$ A. Isaeva, ${ }^{11}$ T. Doert, ${ }^{11}$ \\ L. Hozoi, ${ }^{1}$ A. U. B. Wolter, ${ }^{1}$ B. Büchner, ${ }^{1,5}$ J. Geck, ${ }^{5}$ and J. van den Brink ${ }^{1,12,13}$ \\ ${ }^{1}$ Leibniz-Institut für Festkörper- und Werkstoffforschung (IFW) Dresden, 01171 Dresden, Germany \\ ${ }^{2}$ European Synchrotron Radiation Facility, 38043 Grenoble, France \\ ${ }^{3}$ Institut Laue-Langevin, 38042 Grenoble, France \\ ${ }^{4}$ Instituto de Ciencia de Materiales de Aragón (ICMA), CSIC and Universidad de Zaragoza, 50009 Zaragoza, Spain \\ ${ }^{5}$ Institut für Festkörper- und Materialphysik, Technische Universität Dresden, 01062 Dresden, Germany \\ ${ }^{6}$ IFEG, CONICET, Medina Allende s/n, Ciudad Universitaria, 5000 Cordoba, Argentina \\ ${ }^{7}$ Dresden Center for Computational Materials Science (DCMS), Technische Universität Dresden, 01062 Dresden, Germany \\ ${ }^{8}$ Materials Science and Technology Division, Oak Ridge National Laboratory, Oak Ridge, Tennessee 37831, USA \\ ${ }^{9}$ Department of Materials Science and Engineering, University of Tennessee, Knoxville, Tennessee 37996, USA \\ ${ }^{10}$ Neutron Scattering Division, Oak Ridge National Laboratory, Oak Ridge, Tennessee 37831, USA \\ ${ }^{11}$ Fakultät für Chemie und Lebensmittelchemie, Technische Universität Dresden, 01062 Dresden, Germany \\ ${ }^{12}$ Institut für Theoretische Physik, Technische Universität Dresden, 01062 Dresden, Germany \\ ${ }^{13}$ Department of Physics, Harvard University, Cambridge, Massachusetts 02138, USA
}

(Received 26 January 2018; published 15 June 2018)

\begin{abstract}
Magnetization and high-resolution x-ray diffraction measurements of the Kitaev-Heisenberg material $\alpha-\mathrm{RuCl}_{3}$ reveal a pressure-induced crystallographic and magnetic phase transition at a hydrostatic pressure of $p \sim 0.2 \mathrm{GPa}$. This structural transition into a triclinic phase is characterized by a very strong dimerization of the Ru-Ru bonds, accompanied by a collapse of the magnetic susceptibility. Ab initio quantum-chemistry calculations disclose a pressure-induced enhancement of the direct $4 d-4 d$ bonding on particular Ru-Ru links, causing a sharp increase of the antiferromagnetic exchange interactions. These combined experimental and computational data show that the Kitaev spin-liquid phase in $\alpha-\mathrm{RuCl}_{3}$ strongly competes with the crystallization of spin singlets into a valence bond solid.
\end{abstract}

DOI: 10.1103/PhysRevB.97.241108

The Kitaev model on a honeycomb lattice has grown into a hot topic in the last decade due to its exact solubility and its quantum spin-liquid ground state, which would be relevant for, e.g., quantum computing [1,2]. It implies a bonddependent compass-type coupling $K$ and strong intrinsic spin frustration [3]. A crucial ingredient for realizing the Kitaev model in real materials is a strong spin-orbit coupling together with a honeycomb structure. Recently, Kitaev interactions were identified in $\alpha-\mathrm{RuCl}_{3}$, from its unusual magnetic excitation spectrum [4,5], its strong magnetic anisotropy [6], and electronic-structure calculations $[7,8]$, which render this material an ideal platform for exploring Kitaev magnetism experimentally.

$\alpha-\mathrm{RuCl}_{3}$ is a $j_{\text {eff }}=1 / 2$ Mott insulator with a twodimensional (2D) layered structure of edge-sharing $\mathrm{RuCl}_{6}$ octahedra forming a honeycomb lattice. At ambient pressure,

\footnotetext{
*g.bastien@ifw-dresden.de
}

Published by the American Physical Society under the terms of the Creative Commons Attribution 4.0 International license. Further distribution of this work must maintain attribution to the author(s) and the published article's title, journal citation, and DOI. the honeycomb layers are arranged in a monoclinic $(\mathrm{C} 2 / \mathrm{m})$ structure at room temperature with one of the three nearestneighbor (NN) Ru-Ru bonds slightly shorter than the other two [9]. A structural phase transition was reported at $T_{S} \simeq$ $60 \mathrm{~K}$ under cooling and $T_{S} \bumpeq 166 \mathrm{~K}$ upon warming, but the low-temperature crystal structure is still under debate and could be either rhombohedral $(R \overline{3})[10,11]$ or monoclinic $(C 2 / m)[12,13]$. The onset of long-range magnetic order at $T_{N} \simeq 7 \mathrm{~K}$ [9] in $\alpha-\mathrm{RuCl}_{3}$ implies that other magnetic interactions have to be considered in addition to the Kitaev interaction $K$ : a NN Heisenberg $J$, an off-diagonal coupling $\Gamma$, as well as next-NN interactions $J_{2}$ and $J_{3}[7,8,14,15]$. While electronic-structure calculations indicate that $K$ is ferromagnetic in $\alpha-\mathrm{RuCl}_{3}$ and indeed defines the largest exchange energy scale $[7,8,14,15]$, the debate on the minimal effective spin model and precise magnitude of the different couplings is not fully settled yet. By applying a magnetic field in the basal plane, the magnetic zigzag ground state can be suppressed $[6,16,17]$ and the phase above this transition was identified as a quantum spin liquid, by NMR [18], thermal conductivity [19-21], terahertz spectroscopy [22], and neutron scattering experiments [23].

Further, it was very recently shown by specific heat, magnetization, and NMR measurements [24,25] that the Néel 
temperature of $\alpha-\mathrm{RuCl}_{3}$ increases slightly with pressure and vanishes through a phase separation regime around $0.5 \mathrm{GPa}$ at finite temperature. Thermal expansion measurements at ambient pressure predicted also the suppression of the magnetic order under pressure [26]. However, the initial slope value $d T_{N} / d p_{p=0} \simeq-23 \mathrm{~K} / \mathrm{GPa}$ from thermal expansion is in contradiction with the phase diagram drawn by the other techniques under the application of hydrostatic pressure [24,25]. Magnetization measurements indicate a reduction of the inplane magnetization and a high-temperature transition of unknown origin, while NMR indicates no long-range magnetic order and gapped magnetic fluctuations in the high-pressure state [25]. Furthermore, electrical resistivity studies under hydrostatic pressure exclude the possibility of a pressureinduced insulator-to-metal transition [24].

To clarify the nature of this pressure-induced phase we bring together three essential pieces of information: detailed magnetization and $\mathrm{x}$-ray diffraction measurements on $\alpha-\mathrm{RuCl}_{3}$ under hydrostatic pressure which are combined with a set of quantum-chemistry electronic-structure calculations. Together they unequivocally evidence that pressure induces a first-order structural transition from the rather regular Kitaev-Heisenberg honeycomb system towards a pronounced nonmagnetic dimer state with a large difference between the long and the short RuRu distance of about $0.7 \AA$. Ab initio computations for the highpressure crystal structures reveal remarkably large isotropic antiferromagnetic couplings on the short Ru-Ru bonds, in the range of hundreds of meV, which explain the experimentally observed nonmagnetic state of $\alpha-\mathrm{RuCl}_{3}$. We show that the $j_{\text {eff }}=1 / 2$ picture is significantly modified under hydrostatic pressure as a result of a reduction of spin-orbit-coupling effects due to increased crystal-field splittings in the high-pressure phase.

$\alpha-\mathrm{RuCl}_{3}$ single crystals were grown from phase-pure commercial $\mathrm{RuCl}_{3}$ powder via a high-temperature vapor transport technique $[4,21,27]$. Both magnetization and $\mathrm{x}$-ray diffraction (XRD) show the homogeneous high-quality nature of our single crystals.

Magnetization under hydrostatic pressure was measured in a home-built pressure cell for a commercial superconducting quantum interference device (SQUID) magnetometer from Quantum Design. Two opposing, conical ceramic anvils compress a $\mathrm{CuBe}$ gasket with a small hole that serves as a sample chamber [28]. Daphne oil 7373 is used as a pressure transmitting medium ensuring good hydrostatic conditions up to about $2 \mathrm{GPa}$. The pressure was applied at room temperature and determined at $T \bumpeq 7 \mathrm{~K}$ from the superconducting transition of a lead sample. The magnetic response for the empty cell was measured separately and subtracted from the data, in order to achieve an accuracy on the absolute value of the magnetization of about $2 \times 10^{-3} \mathrm{emu} / \mathrm{mol} / \mathrm{Oe}$ at $\mu_{0} H=1 \mathrm{~T}$.

$\mathrm{X}$-ray diffraction (XRD) experiments as a function of temperature down to $30 \mathrm{~K}$ and pressure up to $11 \mathrm{GPa}$ were performed at the beamline ID27 of the European Synchrotron Radiation Facility, using a monochromatic beam with a photon energy of $33 \mathrm{keV}$ focused down to a spot size of $3 \times 3 \mu \mathrm{m}^{2}$. High-quality single crystals were loaded into a membranedriven diamond anvil cell (DAC) filled with helium as the pressure-transmitting medium. The DAC assembly was then mounted in a continuous He-flow cryostat, allowing one to
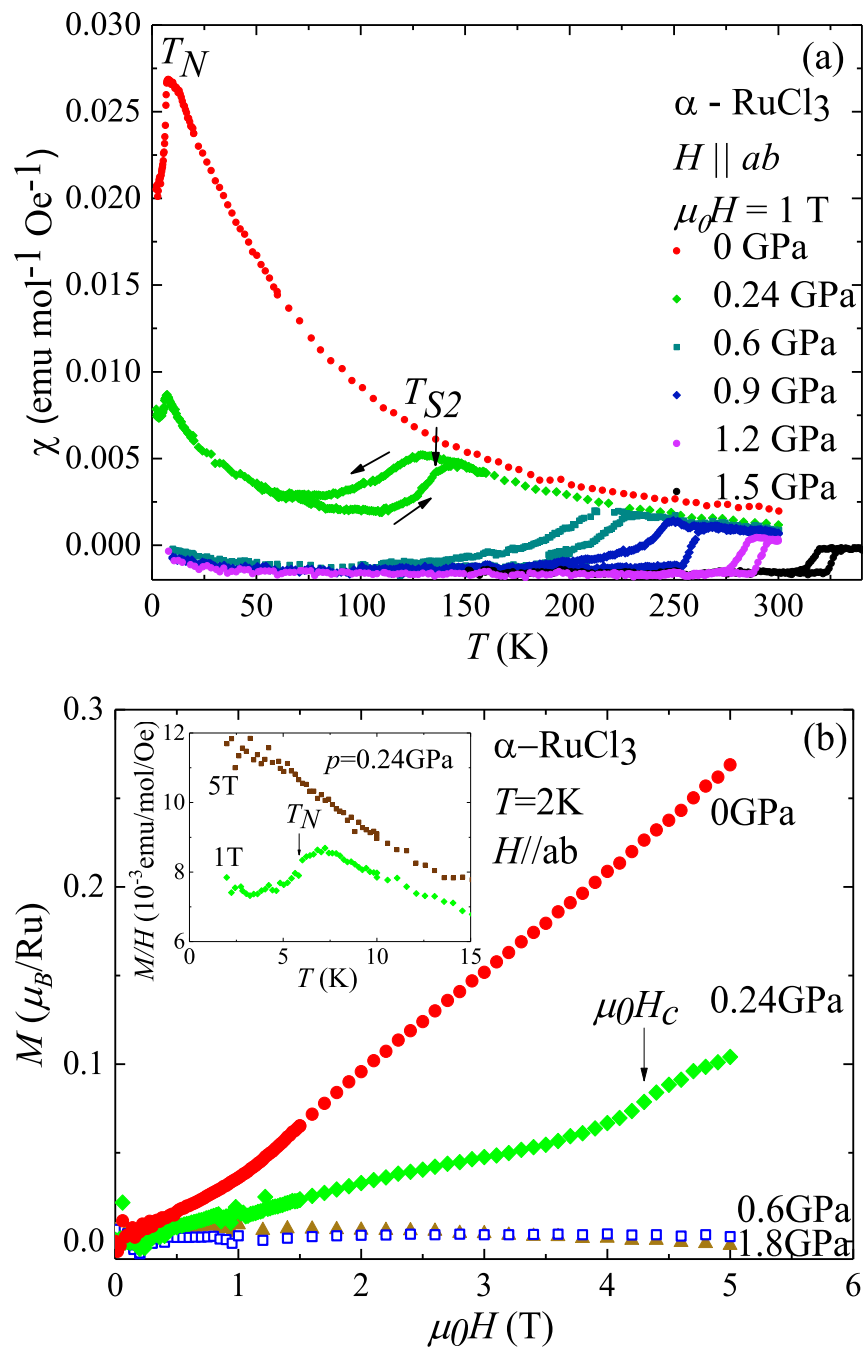

FIG. 1. (a) Field-cooled magnetic susceptibility of $\alpha-\mathrm{RuCl}_{3}$ as a function of temperature for different pressures. A magnetic field of $1 \mathrm{~T}$ was applied in the $a b$ plane. The structural transition $T_{S 2}$ shows thermal hysteresis, and the cooling and warming curves are indicated by black arrows around the $0.24 \mathrm{GPa}$ curve. (b) Magnetization of $\alpha-\mathrm{RuCl}_{3}$ at $2 \mathrm{~K}$ as a function of magnetic field applied in the $a b$ plane for different pressures. $H_{c}$ indicates the phase transition from the zigzag order towards the field-induced quantum spin liquid. The inset shows the renormalized magnetization $M / H$ at $p=0.24 \mathrm{GPa}$ as a function of temperature for magnetic fields of $\mu_{0} H=1 \mathrm{~T}$ and $\mu_{0} H=$ 5 T. $T_{N}$ indicates the magnetic phase transition from the zigzag order to the paramagnetic state.

cool the sample while continuously monitoring the pressure in the sample space via ruby fluorescence. Additional experiments at ID27 without a DAC, i.e., at ambient pressure, were performed as well. The collected three-dimensional data were integrated and corrected for Lorentz, polarization, and background effects using the CRYSALIS PRO software [29]. The subsequent weighted full-matrix least-squares refinements on $F^{2}$ were done with SHELX-2012 [30] as implemented in the WINGX 2014.1 program suite [31].

The magnetic susceptibility $\chi$ of $\alpha-\mathrm{RuCl}_{3}$ in the $a b$ plane is presented in Fig. 1 as a function of $T$ and for different pressures up to $1.5 \mathrm{GPa}$. At ambient pressure the antiferromagnetic 
transition into the zigzag ordered ground state is clearly observable at $T_{N} \simeq 7 \mathrm{~K}$. Under a small hydrostatic pressure of $0.24 \mathrm{GPa}$, a second phase transition occurs at $T_{S 2}=140 \mathrm{~K}$ with a reduction of the magnetic susceptibility by about a factor of 2. This transition shows thermal hysteresis, indicating its first-order structural nature. At $0.6 \mathrm{GPa}$ and higher pressures the transition is shifted to higher temperatures, followed by a strong suppression of the magnetic susceptibility below $T_{S 2}$. Note that the measurement at $0.24 \mathrm{GPa}$ can be interpreted as a phase separation in the sample: While a fraction of the sample is in the high-pressure state and yields a magnetization close to zero below $T_{S 2}$, the second fraction still shows a paramagnetic behavior down to $T_{N} \simeq 7 \mathrm{~K}$, followed by an antiferromagnetic zigzag state below $T_{N}$. These results are qualitatively in good agreement with independent magnetization measurements [25]. While the authors in Ref. [25] measured the magnetic susceptibility on a single crystal with a single Néel temperature $T_{N} \simeq 13.6 \mathrm{~K}$, indicating an $A B$ stacking of the honeycomb layers [9], the measurements reported here were performed on a single crystal with an $A B C$ stacking, as indicated by $T_{N} \simeq 7 \mathrm{~K}$ [9]. The pressure-induced collapse of the in-plane magnetization in Ref. [25] with an $A B$ stacking seems shifted to higher pressure compared to the one reported here and shows a phase separation regime on a broader pressure range up to at least $p \simeq 1 \mathrm{GPa}$. This difference suggests that the stacking sequence would have a small influence on the structural transition $T_{S 2}$.

In order to obtain a deeper insight into the pressureinduced magnetic ground state of $\alpha-\mathrm{RuCl}_{3}$, we performed additional measurements of $\chi$ along the transverse axis $c^{*}$ under hydrostatic pressure, which confirm the collapse of the magnetic susceptibility below $T_{S 2}$ and thus the nonmagnetic nature of the high-pressure state of $\alpha-\mathrm{RuCl}_{3}$ (cf. Supplemental Material [32]).

The magnetization at $2 \mathrm{~K}$ as a function of the magnetic field applied in the basal plane is represented in Fig. 1(b). The magnetization at $p=0.24 \mathrm{GPa}$ shows an upward step at $\mu_{0} H_{c}=4.3 \mathrm{~T}$. Since the temperature scan at $5 \mathrm{~T}$ represented in the inset of Fig. 1(b) confirms the absence of the antiferromagnetic transition above $H_{c}$, the critical field $H_{c}$ corresponds to the suppression of the zigzag order by an external magnetic field similar to $\mu_{0} H_{c} \bumpeq 7-8 \mathrm{~T}$ at ambient pressure $[6,17,18]$. Thus, the quantum critical point toward the field-induced quantum spin-liquid state seems to be strongly reduced from its ambient pressure value in this regime. At even higher pressures of 0.6 and $1.8 \mathrm{GPa}$ a collapse of the magnetic signal is observed in the (pure) high-pressure state up to $5 \mathrm{~T}$, preventing any extraction of the magnetic susceptibility on an absolute scale within the accuracy of our experimental setup.

The resulting temperature-pressure $(T-p)$ phase diagram of $\alpha-\mathrm{RuCl}_{3}$ is given in Fig. 2. The Néel temperature stays rather constant up to about $0.2 \mathrm{GPa}$. Then, $\alpha-\mathrm{RuCl}_{3}$ undergoes a pressure-induced phase transition around $0.2 \mathrm{GPa}$ into a nonmagnetic state with phase separation occurring over a finite pressure range. The transition temperature $T_{S 2}$ increases rapidly with pressure and reaches room temperature around $p=1.3 \mathrm{GPa}$. This phase diagram is in good agreement with previous studies under hydrostatic pressure [24,25] and further shows that the pressure-induced transition is of a first-order nature.

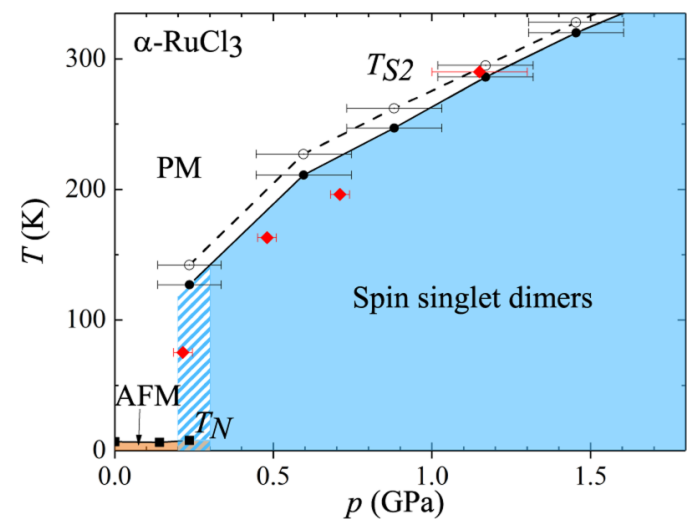

FIG. 2. Temperature-pressure phase diagram of $\alpha-\mathrm{RuCl}_{3}$. The solid and open black circles represent the transition $T_{S 2}$ in magnetization by cooling and by warming, respectively. The red squares represent the transition $T_{S 2}$ from x-ray diffraction. The striped area represents the region where phase separation occurs. The error bars on pressure for the magnetization measurements come from the thermal expansion of the pressure cell. The lines are guides to the eye.

In order to elucidate the microscopic mechanisms underlying the pressure-induced transition at $T_{S 2}$, we performed high-resolution XRD under hydrostatic pressure. At ambient pressure and ambient temperature our XRD measurements confirm the monoclinic $C 2 / m$ structure reported earlier $[9,32]$. Upon increasing pressure, however, a transition $T_{S 2}$ into a triclinic $P \overline{1}$ phase with Ru-Ru dimers was observed together with the changes observed in the magnetic susceptibility as shown in the phase diagram given in Fig. 2. The slight difference between the points from magnetization and from $x$-ray diffraction in this phase diagram can be explained by uncertainties regarding the pressure of the magnetization measurements, by finite transition widths, and by small sample dependencies [27]. The triclinic phase with $\mathrm{Ru}-\mathrm{Ru}$ dimers is stable up to the highest applied pressure of $11 \mathrm{GPa}$.

In order to determine the structural changes in more detail, we performed refinements of the measured intensities at various pressures. The obtained structural changes upon entering the triclinic high-pressure phase are illustrated in Fig. 3. Besides changes in the relative positions of neighboring $\mathrm{RuCl}_{3}$ layers, there are dramatic changes within the layers themselves. At ambient pressure (left panel of Fig. 3), the differences in the Ru-Ru distances are only about $0.003 \AA$ (Table I of Supplemental Material [32]), resulting in a nearly hexagonal honeycomb lattice. The transformation into the triclinic phase with increasing pressure involves the formation of Ru-Ru dimers with a large difference between the short and

TABLE I. NN magnetic couplings (meV) for high-pressure crystal structures as determined at room temperature; results of spin-orbit MRCI calculations for the longer Ru-Ru links, where the isotropic and anisotropic components still have comparable strength.

\begin{tabular}{lcccc}
\hline \hline Pressure (GPa) & $K$ & $J$ & $\Gamma_{x y}$ & $\Gamma_{z x}=-\Gamma_{y z}$ \\
\hline 4.60 & -3.15 & 3.32 & -0.22 & -0.95 \\
10.60 & -1.75 & 0.81 & 0.80 & -0.49 \\
\hline \hline
\end{tabular}




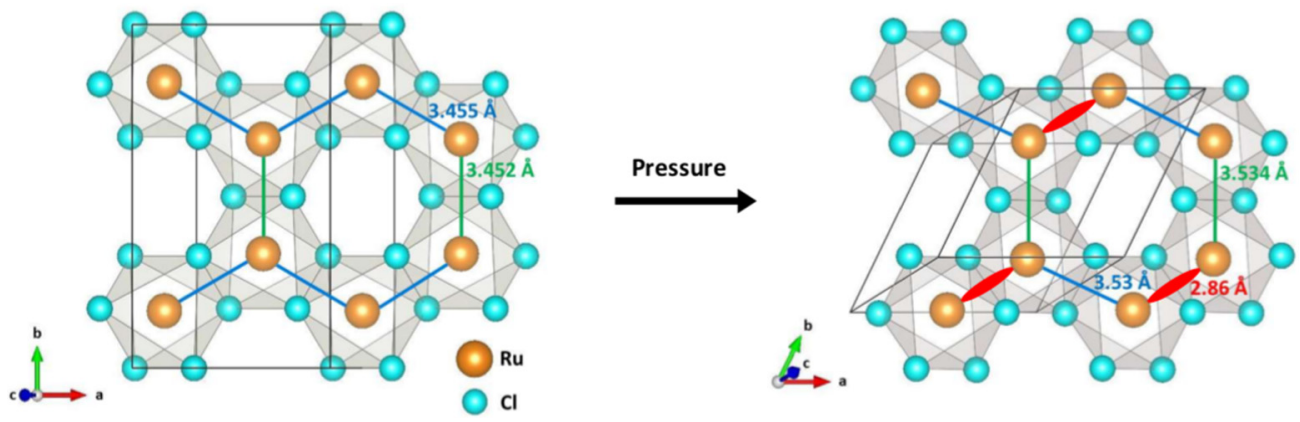

FIG. 3. Honeycomb layer of the $\alpha-\mathrm{RuCl}_{3}$ structure at $300 \mathrm{~K}$ in the monoclinic phase at ambient pressure (left) and in the triclinic phase at $2.08 \mathrm{GPa}$ (right). The ellipses represent the pressure-induced $\mathrm{Ru}-\mathrm{Ru}$ dimers.

the long Ru-Ru distances of about $0.7 \AA$. This extremely strong dimerization involves all $\mathrm{Ru}$ atoms, i.e., every $\mathrm{Ru}$ atom is part of a dimer.

To clarify the effect of this dimerization on magnetism, we carried out embedded-cluster quantum-chemistry calculations using the experimental crystal structures. Our ab initio results show that in the triclinic phase the $4 d$-shell $t_{2 g}$ crystal-field splittings are very large, up to $0.35 \mathrm{eV}$, and counteract the effect of spin-orbit coupling. The $j_{\text {eff }}=1 / 2$ picture is therefore significantly modified and given the peculiar character of the $\mathrm{Ru} t_{2 g}$ hole, an antiferromagnetic isotropic spin model turns out to be a rather good approximation on the shortest $\mathrm{Ru}-\mathrm{Ru}$ links, with an impressively strong antiferromagnetic exchange. Specifically, we find that two of the $4 d t_{2 g}$ levels are nearly degenerate, lie at lower energy (electron picture), and that the $t_{2 g}$ hole is mainly associated with the high-energy orbital that provides a large direct $d$ - $d$ overlap on the shortest Ru-Ru bonds, as sketched in Fig. 4. By multireference configurationinteraction (MRCI) calculations [33] we derive singlet-triplet

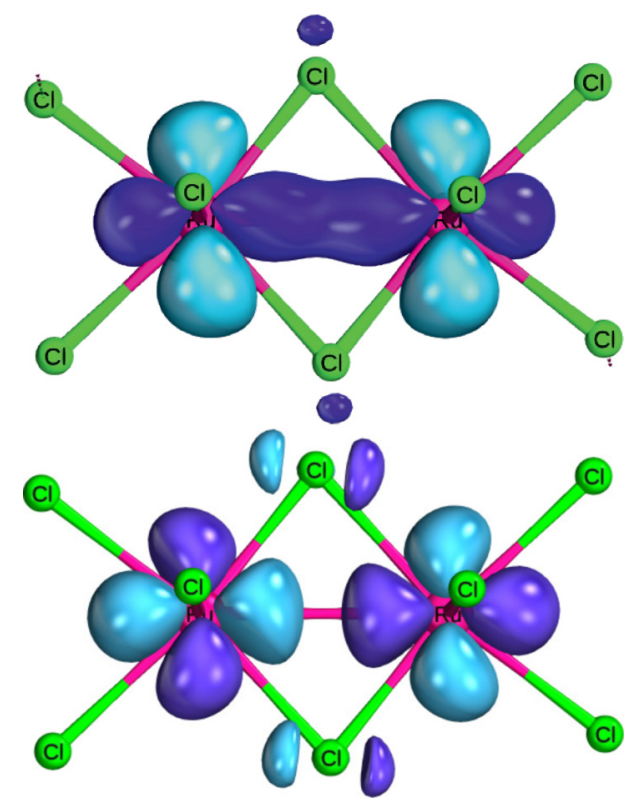

FIG. 4. Bonding (top) and antibonding (bottom) combinations of the $\mathrm{Ru} t_{2 g}$ hole orbitals on the shorter $\mathrm{Ru}-\mathrm{Ru}$ bonds of the crystal structure in the dimer state, as obtained by embedded-cluster quantum-chemistry calculations. separation energies as high as 440 and $550 \mathrm{meV}$ for the shortest $\mathrm{Ru}-\mathrm{Ru}$ links found experimentally at $300 \mathrm{~K}$ for 4.6 and $10.6 \mathrm{GPa}$, respectively, with vanishing splittings among the triplet components. So large energy differences between the singlet and triplet states associated with two $\mathrm{NN} t_{2 g}^{5}$ ions imply that a finite magnetization can only be achieved by very large magnetic fields, which is indeed observed in Fig. 1(b), and also explain the large spin-excitation gap observed in a recent NMR study of $\alpha-\mathrm{RuCl}_{3}$ under pressure [25].

For the longer Ru-Ru links, the relevant effective model is an extended pseudospin-1/2 Hamiltonian with both isotropic and anisotropic components [8],

$$
\mathcal{H}_{i, j}=J \tilde{\mathbf{S}}_{i} \cdot \tilde{\mathbf{S}}_{j}+K \tilde{S}_{i}^{z} \tilde{S}_{j}^{z}+\sum_{\alpha \neq \beta} \Gamma_{\alpha \beta}\left(\tilde{S}_{i}^{\alpha} \tilde{S}_{j}^{\beta}+\tilde{S}_{i}^{\beta} \tilde{S}_{j}^{\alpha}\right)
$$

where $\tilde{\mathbf{S}}_{i}$ and $\tilde{\mathbf{S}}_{j}$ are NN pseudospin $1 / 2$ operators and the $\Gamma_{\alpha \beta}$ coefficients stand for off-diagonal couplings of the anisotropic exchange tensor with $\alpha, \beta \in\{x, y, z\}$. Mapping the spin-orbit MRCI results onto such an effective model [8,34], we arrive at the NN couplings listed in Table I for the long links of the dimerized structure at 4.6 and $10.6 \mathrm{GPa}$.

The combined experimental and theoretical results therefore reveal a competition between spin-orbit coupling and covalency effects. Interestingly, a second crystal structure, $\beta-\mathrm{RuCl}_{3}$, with $\mathrm{Ru}$ chains at room temperature and $\mathrm{Ru}$ dimers at low temperature, was reported [35]. Previous densityfunctional calculations predicted that $\alpha-\mathrm{RuCl}_{3}$ would also dimerize in the absence of spin-orbit coupling [36]. While at ambient pressure the spin-orbit coupling is significantly larger than the crystal-field splittings to stabilize a $\mathrm{Ru} j_{\text {eff }} \simeq$ $1 / 2$ state, with increasing pressure a phase dominated by strong covalency appears [37-39]. This mechanism may also apply to other $4 d$ and $5 d$ metal halides and oxides such as $\alpha-\mathrm{MoCl}_{3}$ at ambient pressure [40,41] and the KitaevHeisenberg iridate $\alpha-\mathrm{Li}_{2} \mathrm{IrO}_{3}$ at a critical pressure $p_{c} \simeq$ $3.8 \mathrm{GPa}$ [42]. Thus dimerization may be a rather general feature of $4 d$ and $5 d$ honeycomb systems, due to a subtle interplay between spin-orbit coupling, intermetallic bonding, and magnetism.

In summary, our magnetization and x-ray diffraction experiments on $\alpha-\mathrm{RuCl}_{3}$ under hydrostatic pressure show a pressure-induced phase transition from the monoclinic to a 
triclinic structure, featuring a very pronounced $\mathrm{Ru}-\mathrm{Ru}$ dimerization and a valence bond crystal of ordered dimers. The latter are characterized by remarkably strong antiferromagnetic isotropic couplings due to an increased direct overlap of the $\mathrm{Ru} 4 d t_{2 g}$ orbitals. This dimerization leads to a complete suppression of the magnetization and thus to a pressureinduced nonmagnetic state of $\alpha-\mathrm{RuCl}_{3}$. Our results show that the Kitaev physics in this $d$-electron honeycomb system is in competition with the formation of spin singlet valence bonds: Indeed, $\alpha-\mathrm{RuCl}_{3}$ shows the occurrence of both a quantum spin-liquid state under magnetic field, which is relevant for its topological properties, and a spin solid under hydrostatic pressure, the spin singlet valence bond crystal. Thus, this material will provide insights for the study of concomitant magnetic and lattice instabilities in $4 d$ and $5 d$ metal halides and oxides.
We acknowledge insightful discussions with Manuel Richter, Matthias Vojta, Lukas Janssen, and Hans-Henning Klauss. This research has been supported by the DFG via SFB 1143 and WO 1532/3-2 and by the European Union's Horizon 2020 research and innovation programme under the Marie Sklodowska-Curie Grant Agreement No 796048. M. Mezouar is acknowledged for providing beamtime at ID27 at the ESRF and for fruitful discussions. S.P.L. is grateful to CONICET for financial support during her stay at the ESRF. R.Y. and L.H. acknowledge Ulrike Nitzsche for technical support as concerns the $a b$ initio calculations. P.L.K and D.G.M. were supported by the Gordon and Betty Moore Foundation EPiQS Initiative Grant No. GBMF4416. S.N. was supported by the U.S. Department of Energy, Basic Energy Sciences, Scientific User Facilities Division under contract DE-AC0500OR22725 with the Oak Ridge National Laboratory.
[1] J. Knolle, D. L. Kovrizhin, J. T. Chalker, and R. Moessner, Phys. Rev. B 92, 115127 (2015).

[2] S.-H. Do, S.-Y. Park, J. Yoshitake, J. Nasu, Y. Motome, Y. Kwon, D. T. Adroja, D. J. Voneshen, K. Kim, T.-H. Jang, J.-H. Park, K.-Y. Choi, and S. Ji, Nat. Phys. 13, 1079 (2017).

[3] A. Kitaev, Ann. Phys. 321, 2 (2006).

[4] A. Banerjee, C. A. Bridges, J.-Q. Yan, A. A. Aczel, L. Li, M. B. Stone, G. E. Granroth, M. D. Lumsden, Y. Yiu, J. Knolle, S. Bhattacharjee, D. L. Kovrizhin, R. Moessner, D. A. Tennant, D. G. Mandrus, and S. E. Nagler, Nat. Mater. 15, 733 (2016).

[5] A. Banerjee, J. Yan, J. Knolle, C. A. Bridges, M. B. Stone, M. D. Lumsden, D. G. Mandrus, D. A. Tennant, R. Moessner, and S. E. Nagler, Science 356, 1055 (2017).

[6] M. Majumder, M. Schmidt, H. Rosner, A. A. Tsirlin, H. Yasuoka, and M. Baenitz, Phys. Rev. B 91, 180401 (2015).

[7] S. M. Winter, Y. Li, H. O. Jeschke, and R. Valentí, Phys. Rev. B 93, 214431 (2016).

[8] R. Yadav, N. A. Bogdanov, V. M. Katukuri, S. Nishimoto, J. van den Brink, and L. Hozoi, Sci. Rep. 6, 37925 (2016).

[9] H. B. Cao, A. Banerjee, J.-Q. Yan, C. A. Bridges, M. D. Lumsden, D. G. Mandrus, D. A. Tennant, B. C. Chakoumakos, and S. E. Nagler, Phys. Rev. B 93, 134423 (2016).

[10] S.-Y. Park, S.-H. Do, K.-Y. Choi, D. Jang, T.-H. Jang, J. Schefer, C.-M. Wu, J. S. Gardner, J. M. S. Park, J.-H. Park, and S. Ji, arXiv:1609.05690.

[11] S. Reschke, F. Mayr, S. Widmann, H.-A. Krug von Nidda, V. Tsurkan, M. V. Eremin, S.-H. Do, K.-Y. Choi, Z. Wang, and A. Loidl, arXiv:1803.04887.

[12] A. Little, L. Wu, P. Lampen-Kelley, A. Banerjee, S. Patankar, D. Rees, C. A. Bridges, J.-Q. Yan, D. Mandrus, S. E. Nagler, and J. Orenstein, Phys. Rev. Lett. 119, 227201 (2017).

[13] P. Lampen-Kelley, S. Rachel, J. Reuther, J.-Q. Yan, A. Banerjee, C. A. Bridges, H. B. Cao, S. E. Nagler, and D. Mandrus, arXiv:1803.04871.

[14] L. Janssen, E. C. Andrade, and M. Vojta, Phys. Rev. B 96, 064430 (2017).

[15] S. M. Winter, K. Riedl, D. Kaib, R. Coldea, and R. Valentí, Phys. Rev. Lett. 120, 077203 (2018).

[16] J. A. Sears, Y. Zhao, Z. Xu, J. W. Lynn, and Y.-J. Kim, Phys. Rev. B 95, 180411(R) (2017).
[17] A. U. B. Wolter, L. T. Corredor, L. Janssen, K. Nenkov, S. Schönecker, S.-H. Do, K.-Y. Choi, R. Albrecht, J. Hunger, T. Doert, M. Vojta, and B. Büchner, Phys. Rev. B 96, 041405(R) (2017).

[18] S.-H. Baek, S.-H. Do, K.-Y. Choi, Y. S. Kwon, A. U. B. Wolter, S. Nishimoto, J. van den Brink, and B. Büchner, Phys. Rev. Lett. 119, 037201 (2017).

[19] I. A. Leahy, C. A. Pocs, P. E. Siegfried, D. Graf, S.-H. Do, K.-Y. Choi, B. Normand, and M. Lee, Phys. Rev. Lett. 118, 187203 (2017).

[20] Y. J. Yu, Y. Xu, K. J. Ran, J. M. Ni, Y. Y. Huang, J. H. Wang, J. S. Wen, and S. Y. Li, Phys. Rev. Lett. 120, 067202 (2018).

[21] R. Hentrich, A. U. B. Wolter, X. Zotos, W. Brenig, D. Nowak, A. Isaeva, T. Doert, A. Banerjee, P. Lampen-Kelley, D. G. Mandrus, S. E. Nagler, J. Sears, Y.-J. Kim, B. Büchner, and C. Hess, Phys. Rev. Lett. 120, 117204 (2018).

[22] Z. Wang, S. Reschke, D. Hüvonen, S.-H. Do, K.-Y. Choi, M. Gensch, U. Nagel, T. Rõõm, and A. Loidl, Phys. Rev. Lett. 119, 227202 (2017).

[23] A. Banerjee, P. Lampen-Kelley, J. Knolle, C. Balz, A. A. Aczel, B. Winn, Y. Liu, D. Pajerowski, J. Yan, C. A. Bridges, A. T. Savici, B. C. Chakoumakos, M. D. Lumsden, D. A. Tennant, R. Moessner, D. G. Mandrus, and S. E. Nagler, npj Quantum Mater. 3, 8 (2018).

[24] Z. Wang, J. Guo, F. F. Tafti, A. Hegg, S. Sen, V. A. Sidorov, L. Wang, S. Cai, W. Yi, Y. Zhou, H. Wang, S. Zhang, K. Yang, A. Li, X. Li, Y.Li, J. Liu, Y. Shi, W. Ku, Q. Wu et al., arXiv:1705.06139.

[25] Y. Cui, J. Zheng, K. Ran, J. Wen, Z.-X. Liu, B. Liu, W. Guo, and W. Yu, Phys. Rev. B 96, 205147 (2017).

[26] M. He, X. Wang, L. Wang, F. Hardy, T. Wolf, P. Adelmann, T. Brückel, Y. Su, and C. Meingast, arXiv:1712.08511.

[27] The single crystals for the magnetization studies were grown at the Oak Ridge National Laboratory, while the single crystals for the X-ray diffraction experiments were grown at the TU Dresden.

[28] N. Tateiwa, Y. Haga, Z. Fisk, and Y. Ōnuki, Rev. Sci. Instrum. 82, 053906 (2011).

[29] CRYSALIS PRO Software System 1.171.38.43, Rigaku Oxford Diffraction, 2016.

[30] G. M. Sheldrick, Acta Crystallogr., Sect. A: Found. Crystallogr. 64, 112 (2007). 
[31] L. J. Farrugia, J. Appl. Crystallogr. 32, 837 (1999).

[32] See Supplemental Material at http://link.aps.org/supplemental/ 10.1103/PhysRevB.97.241108 for a magnetization study along the transverse axis $c^{*}$, reversibility studies of the pressure dependence, the structural refinement under pressure, and computational details on the $a b$ initio quantum-chemistry calculations, which includes Refs. [43-48].

[33] T. Helgaker, P. Jørgensen, and J. Olsen, Molecular ElectronicStructure Theory (Wiley, Chichester, 2000).

[34] N. A. Bogdanov, V. M. Katukuri, J. Romhányi, V. Yushankhai, V. Kataev, B. Büchner, J. van den Brink, and L. Hozoi, Nat. Commun. 6, 7306 (2015).

[35] H. Hillebrecht, T. Ludwig, and G. Thiele, Z. Anorg. Allg. Chem. 630, 2199 (2004).

[36] H.-S. Kim and H.-Y. Kee, Phys. Rev. B 93, 155143 (2016).

[37] G. Jackeli and D. A. Ivanov, Phys. Rev. B 76, 132407 (2007).

[38] G. Jackeli and D. I. Khomskii, Phys. Rev. Lett. 100, 147203 (2008).

[39] S. V. Streltsov and D. I. Khomskii, Proc. Natl. Acad. Sci. USA 113, 10491 (2016).
[40] H. Schäfer, H.-G. V. Schnering, J. Tillack, F. Kuhnen, H. Wöhrle, and H. Baumann, Z. Anorg. Allg. Chem. 353, 281 (1967).

[41] M. A. McGuire, J. Yan, P. Lampen-Kelley, A. F. May, V. R. Cooper, L. Lindsay, A. Puretzky, L. Liang, S. KC, E. Cakmak, S. Calder, and B. C. Sales, Phys. Rev. Mater. 1, 064001 (2017).

[42] V. Hermann, M. Altmeyer, J. Ebad-Allah, F. Freund, A. Jesche, A. A. Tsirlin, M. Hanfland, P. Gegenwart, I. I. Mazin, D. I. Khomskii, R. Valentí, and C. A. Kuntscher, Phys. Rev. B 97, 020104 (2018).

[43] H. J. Werner, P. J. Knowles, G. Knizia, F. R. Manby, and M. Schütz, Wiley Interdiscip. Rev.: Comput. Mol. Sci. 2, 242 (2012).

[44] M. Klintenberg, S. Derenzo, and M. Weber, Comput. Phys. Commun. 131, 120 (2000).

[45] K. A. Peterson, D. Figgen, M. Dolg, and H. Stoll, J. Chem. Phys. 126, 124101 (2007).

[46] D. E. Woon and T. H. Dunning, J. Chem. Phys. 98, 1358 (1993).

[47] K. Pierloot, B. Dumez, P.-O. Widmark, and B. O. Roos, Theor. Chim. Acta 90, 87 (1995).

[48] A. Berning, M. Schweizer, H.-J. Werner, P. J. Knowles, and P. Palmieri, Mol. Phys. 98, 1823 (2000). 